\title{
Review
}

\section{Innovative low-glycaemic carbohydrates: an update} How a smart choice can contribute to a healthier life

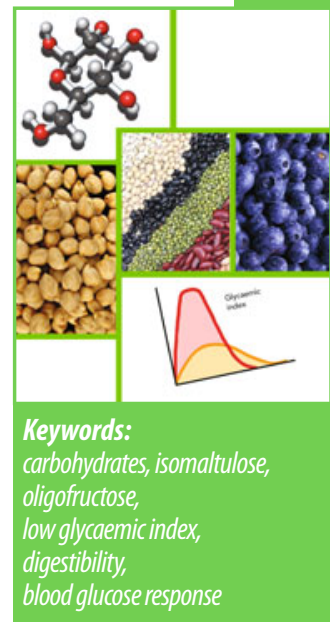

Received: 30 October 2013 / Accepted: 9 December 2013

(C) Springer Healthcare - CEC Editore 2013

\section{Abstract}

The key role of carbohydrates in nutrition is to deliver energy to the body. Until recently, debates on preferred types of carbohydrates as opposed to those that should be avoided focused mainly on a simple food chemistry approach. This approach does not reflect physiology, although it is used for food labelling and analysis purposes. It is the metabolism of carbohydrates that counts when we consider the increasing burden of non-communicable diseases. Since carbohydrates are the largest macronutrient category, with a recommended intake of 55\%-75\%, their influence is significant. Digestibility, availability, speed of absorption and metabolic pathways are key. It is the quality of carbohydrates that matters and a low glycaemic diet that counts. Tasty, lower glycaemic food can be achieved in various ways. Reducing the blood glucose response of available carbohydrates by modification of the glucose supply can be achieved by processing, by influencing the gastrointestinal pas-

\footnotetext{
Anke Sentko $(\bowtie)$

BENEO-Institute c/o BENEO GmbH

Wormser Str. 11

67283 Obrigheim, Germany

tel +496359 803-822

fax +496359803839

Anke.Sentko@BENEO.com
}

sage and by a smart choice of ingredients: by selecting low glycaemic and slow-release carbohydrates. An alternative route is the replacement of available carbohydrates by partially or non-available carbohydrates, such as dietary fibre or traditional sugar replacers.

\section{Introduction}

The key role of carbohydrates in nutrition is to deliver energy to the body, as the body's fuel is glucose. According to the European Food Safety Authority's nutrient intake recommendations, the carbohydrate content in the daily diet should be between $40 \%$ and $60 \%$ of energy intake [1]. On a global basis, the World Health Organization (WHO) postulates that $55 \%-75 \%$ of energy intake should come from carbohydrates, $15 \%-30 \%$ from fat and 10\%-15\% from protein [2].

Until recently, debates on preferred types of carbohydrates as opposed to those which should be avoided focused mainly on a simple food chemistry approach and the belief that "simple" carbohydrates (i.e., mono- and disaccharides) should be reduced and "complex" carbohydrates (this term is often used for carbohydrates from starchy foods and refers to a longer-chain carbohydrate structure [polysaccharides]) are recommended. Although this food chemistry approach is often applied for food 
labelling and food control analytics, it does not meet today's requirements and does not reflect physiology at all. How nutrients are digested and absorbed, how quickly they enter the body, the way they are metabolised, and the way hormones and other biomarkers are influenced by this all play a major role in healthy eating and a healthy lifestyle on the one hand and in the development of non-communicable diseases on the other hand. As carbohydrates are the largest macronutrient category, their influence on metabolism is significant; until recently, however, the attention this nutrient group received from a physiological point of view was minor. Consumers still receive food chemistry messages with a simple differentiation between sugars and starches, if anything. Health-care professionals often avoid communication on the blood glucose effect of food, glycaemic index (GI) and glycaemic load (GL) information, as they consider it too complex for the consumer. Consequently, consumers are still misled by the message that all sugars are bad and all starches are good.

\section{The quality of carbohydrates counts}

A fresh analysis of what is really needed leads to a debate among health-care professionals about the quality of carbohydrates, focussing on the individual physiological properties of various carbohydrates. The top parameters to monitor are digestibility and the influence on the blood glucose profile, as well as the related insulin response, on a shortand long-term basis.

\section{Digestibility of carbohydrates}

The characteristics of the digestibility of a carbohydrate reflect its uptake by the body and consequently influence the blood glucose and insulin response.

The term 'digestibility' generally addresses the digestion process in the human small intestine. To be absorbed, a carbohydrate needs to be broken down into its monosaccharide units. Only monosaccharides can enter the body for metabolism. Enzymes specialising in carbohydrate breakdown are available in large amounts throughout the length of the small intestine.

Figure 1 illustrates the degree of digestibility of a number of carbohydrates that are structured by their chemical classification as sugars, oligosaccharides and polysaccharides. The figure clearly visualises that it is not the chemical structure that determines the degree of digestibility. This becomes even more obvious when looking at some individual postprandial blood glucose response curves and GI/GL tables. Starchy foods like cooked potatoes or steamed rice are immediately available, even though the carbohydrates are of a polysaccharide type. Some other carbohydrates have low digestibility, i.e., they are only partially available in the small intestine or even non-digestible (i.e., not available in the small intestine). Examples for low-digestible carbohydrates are polyols; examples for non-digestible carbohydrates are dietary fibres such as resistant starches or inulin-type fructans (inulin, oligofructose) from chicory. The non-digestible carbohydrates escape digestion and absorption and travel along the small intestine untouched. Most of them are then fermented by the gut microflora in the large intestine. The higher the content of non-digestible parts in a carbohydrate mixture in a food (replacing digestible carbohydrates), the lower the glycaemic response of that food when measuring blood sugar. Whilst the vast majority of carbohydrates are rapidly available and correspondingly high glycaemic, there is one innovative carbohydrate that is fully available in the small intestine but is slowly hydrolysed by the enzymes in the small intestine, i.e., four to five times more slowly, leading to a low glycaemic response. This carbohydrate is isomaltulose (Palatinose ${ }^{\mathrm{TM}}$ ), a disaccharide with a glucose and a fructose unit that are linked by an $\alpha-1,6$-glycosidic binding, which takes more time to be digested. In fact, fully available carbohydrates are high glycaemic; a lower glycaemic profile can only be achieved by increasing the non-digestible part of the total carbohydrate content (= escaping small intestine absorption = 


\begin{tabular}{|c|c|c|c|c|}
\hline & \multicolumn{2}{|c|}{ Sugars (DP 1-2) } & \multirow{2}{*}{$\begin{array}{l}\text { Oligosaccharide } \\
\text { (DP 3-9) }\end{array}$} & \multirow{2}{*}{$\begin{array}{l}\text { Polysaccharide } \\
(\mathrm{DP}>9)\end{array}$} \\
\hline & Monosaccharide & Disaccharide & & \\
\hline & Glucose & Maltose & Malto-oligosaccharides/maltodextrin & $\begin{array}{l}\text { Starch }^{1} \\
\text { - Amylose } \\
\text { - Amylopectin }\end{array}$ \\
\hline & \multirow{2}{*}{ Fructose ${ }^{1}$} & Sucrose & \multirow{2}{*}{ Fructo-oligosaccharides } & \multirow{3}{*}{$\begin{array}{l}\text { Resistant starch } \\
\text { Non-starch polysaccharides: } \\
\text { - Cellulose } \\
\text { - Hemicellulose } \\
\text { - Pectins } \\
\text { - Inulin }\end{array}$} \\
\hline & & Isomaltulose $\left(\right.$ Palatinose $\left.{ }^{\mathrm{TM}}\right)$ & & \\
\hline & Galactose & Lactose $^{1}$ & Galacto-oligosaccharides & \\
\hline \multirow[t]{2}{*}{$\begin{array}{l}\text { Polyols/sugar } \\
\text { replacers }\end{array}$} & $\begin{array}{l}\text { Sorbitol } \\
\text { Mannitol } \\
\text { Xylitol }\end{array}$ & $\begin{array}{l}\text { Isomalt } \\
\text { Maltitol }\end{array}$ & $\begin{array}{l}\text { Maltitol syrup } \\
\text { Hydrogenated Starch Hydrolysate }\end{array}$ & \\
\hline & HIGHLY digestible & SLOWLY and FULLY digestible & LOW-/NON- digestible & \\
\hline
\end{tabular}

Figure 1 The chemical classification classifies carbohydrates according to their degree of polymerisation (DP), i.e., the number of monosaccharide units linked together. It does not reflect the physiological properties of carbohydrates and their effect on blood glucose levels. The digestibility and related physiological properties of those carbohydrates can vary widely within each group, as indicated by the yellow and green colors

loss of available energy and increase in large intestine load for fermentation processes).

The uniqueness of isomaltulose is obvious: its low glycaemic property stems from its slow but complete digestion in the small intestine. Carbohydrate energy from isomaltulose enters the body in a slow, low and sustained way; in total, energy delivery is complete and no carbohydrate load reaches the large intestine (i.e., no gastrointestinal distress occurs).

\section{Improvement of quality of physiological carbohydrates by reducing the blood glucose response of available carbohydrates: \\ modification of glucose supply}

A reduction of the blood glucose response of available carbohydrates by modifying the glucose supply can be achieved by

- specific processing techniques (starch and baking process)

- some viscous soluble fibres when used in high amounts
- a smart choice of low glycaemic but fully available alternatives: isomaltulose

The last option mentioned is the most recent, since isomaltulose was introduced to the $\mathrm{EU}$ market in 2005 after approval as a Novel Food [3]. To build up knowledge about isomaltulose, the emerging physiological profile of this innovative, unique ingredient is described here. It provides real incentives for development of innovative products supporting healthy nutrition.

\section{Isomaltulose: a unique innovative carbohydrate}

\section{The key physiological features in a nutshell}

Isomaltulose is a sugar-type carbohydrate with a unique combination of physiological properties:

- Completely available carbohydrate and glucose supplier (4 kcal/g)

- Slow release of energy

- Low effect on blood glucose (GI: 32) and insulin (insulin index [II]: 30)

- Balanced and prolonged glucose supply 


\begin{tabular}{|c|c|c|}
\hline & Type of key studies & Demonstrated physiological properties \\
\hline Mouth & pH-telemetry & Does not promote dental caries \\
\hline \multicolumn{3}{|l|}{ Small intestine } \\
\hline Hydrolysis & Enzyme kinetics (in vitro) & Slow hydrolysis into glucose+fructose (4-5 times slower than sucrose) \\
\hline \multirow[t]{4}{*}{ Absorption } & lleostomy study & Virtually complete hydrolysis and absorption within the small intestine \\
\hline & Blood glucose response & $\begin{array}{l}\text { Slow increase, low glycaemic response } \\
\text { Glucose (energy) delivery over a prolonged period of time }\end{array}$ \\
\hline & Insulin response & Low insulin response \\
\hline & $\mathrm{Gl}$ & 32 \\
\hline Large intestine & Gastrointestinal tolerance & No distress even at high levels (e.g., $120 \mathrm{~g}$ in a sports study) \\
\hline Metabolism & $\begin{array}{l}\text { Respiratory quotient } \\
\text { Body composition }\end{array}$ & $\begin{array}{l}\text { Promotes fat burning } \\
\text { Loss of body fat and body weight/prevention of weight gain }\end{array}$ \\
\hline
\end{tabular}

Table 1 Physiological properties of isomaltulose

- Higher contribution to fat oxidation in energy metabolism

- Kind to teeth

Table 1 provides the key features of isomaltulose in a brief overview following the pathway of food through the body - from the mouth to the faeces including metabolic aspects.

The low/reduced glycaemic properties of isomaltulose and its potential to reduce the glycaemic response of foods when replacing other sugars (partially or completely) have been confirmed by a positive EFSA opinion [4] and the approval of a corresponding health claim in the European Union, laid down in the Annex of Regulation EC 432/2012. In addition, its toothfriendliness has been confirmed by the FDA approval of a corresponding dental health claim in the US Code of Federal Regulations (21CFR \$101.80) and by a positive EFSA opinion in the EU [4], with a corresponding claim approved in the Annex of Regulation EC 432/2012.

\section{Where isomaltulose comes from}

Isomaltulose occurs naturally in small quantities in honey and sugarcane juices $[5,6]$. On a large scale, isomaltulose is produced by BENEO GmbH, a 100\% subsidiary of Südzucker, from sugar extracted from sugar beet by an enzymatic step (non-GMO) that shifts the bond between the glucose and fructose moiety from an $\alpha$-1-2- into an $\alpha$-1,6-glycosidic bond, which is much stronger and stable against enzymatic or acid influences (see Fig. 2). This is the basis of the key physiological characteristics as well.

\section{Isomaltulose: key physicochemical properties} and stability

Isomaltulose has the following physicochemical properties $[7,8]$ :

- Taste and appearance are similar to sucrose, providing a natural and refreshing perception of sweetness quickly and without aftertaste.

- Its sweetening power, in comparison to a $10 \%$ sucrose solution, is about $50 \%$. Isomaltulose provides a mild sweetness without reducing the volume and the "body" of a food or beverage.

- In an aqueous solution, the viscosity of sucrose and isomaltulose are similar.

- The solubility of isomaltulose is $29 \%\left(20^{\circ} \mathrm{C}\right.$, aqueous solution).

- The melting temperature is lower $\left(120-128^{\circ} \mathrm{C}\right)$ than that of sucrose $\left(160-185^{\circ} \mathrm{C}\right)$.

- It shows very good stability under acidic conditions, for instance in beverages, upon thermal treatment or in a bacterial environment.

- It is not hygroscopic and the powder has excellent flowability.

- Its shelf life is comparable to that of sucrose when stored under dry conditions and moderate temperatures. 


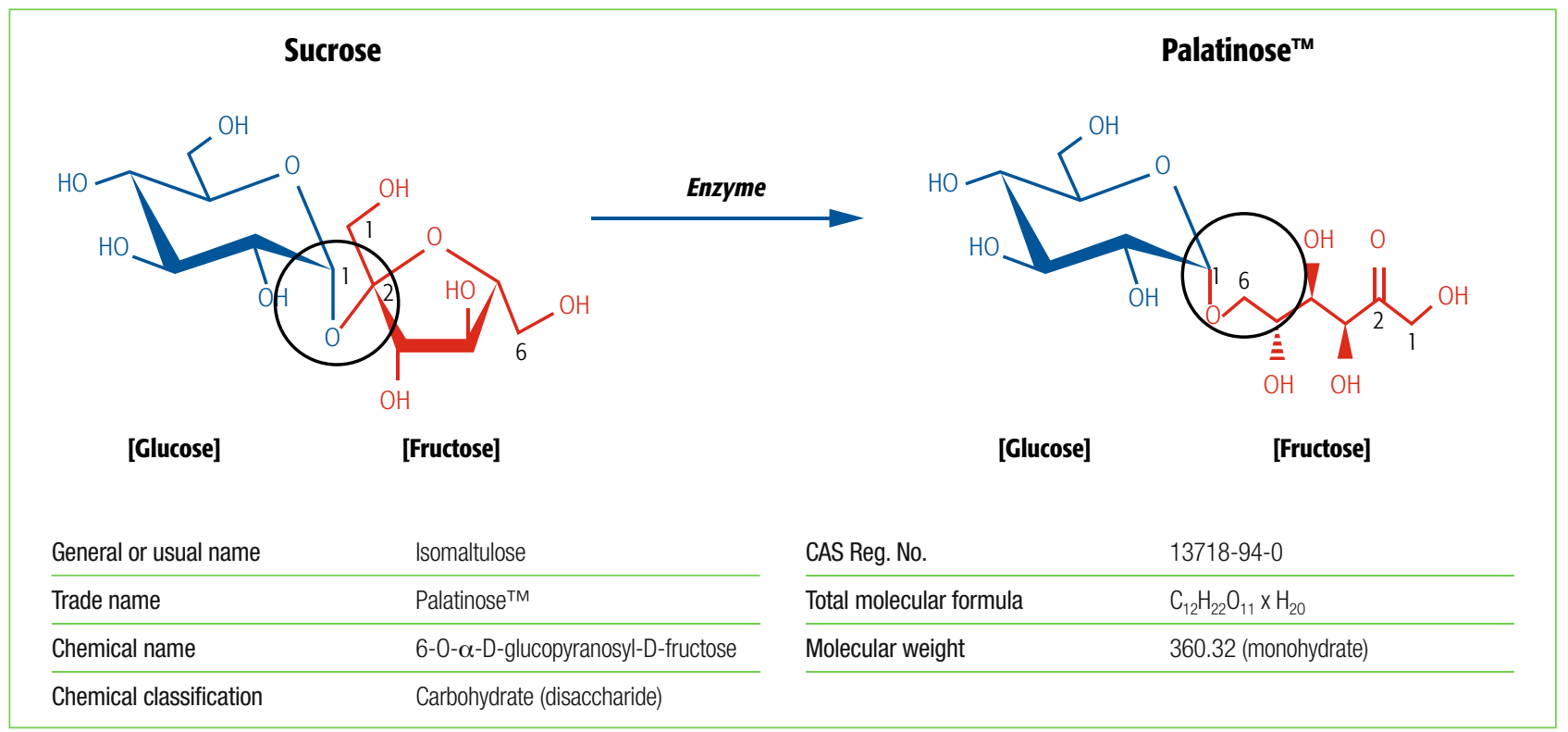

Figure 2 Enzymatic rearrangement of sucrose to Palatinose ${ }^{\mathrm{TM}}$ (isomaltulose)

Isomaltulose has good heat stability upon processing and it is stable in acidic conditions and in a bacterial environment, for instance, in the presence of lactic bacteria in dairy applications. Therefore, isomaltulose is not expected to undergo significant hydrolytic changes on processing or as an ingredient in typical food product applications over the course of time. Under the physiological conditions in the stomach $\left(37^{\circ} \mathrm{C}, 3 \mathrm{~h}, \mathrm{pH} 1\right.$ and 2$)$, isomaltulose showed no hydrolysis, whereas sucrose was degraded to $55 \%$ and 7\%, respectively. The high stability of isomaltulose in food applications and its complete bioavailability to the human body are essential features of the mechanism behind its physiological benefits. Isomaltulose can be used in food products as a low glycaemic carbohydrate source for the purpose of reducing the glycaemic and insulinaemic properties of foods.

Isomaltulose as a slow-release, fully available carbohydrate energy supply in a slower, more balanced way and over a longer period of time than conventional carbohydrates

The slow release and low glycaemic properties of isomaltulose are based on its physiological charac- teristics with respect to digestion, absorption, blood glucose response and subsequent effects on insulin release and metabolic regulation [9]:

- As a disaccharide carbohydrate, isomaltulose requires intestinal digestion prior to the absorption of its monosaccharide constituents into the blood and the body.

- The $\alpha-1,6$ linkage in isomaltulose is more slowly digested by intestinal enzymes than the $\alpha-1,2$ linkage in sucrose (by a factor of about 4-5) or the $\alpha$ 1,4 linkage in maltose-type starch digestion products (by a factor of about 8-10), resulting in a slower release of glucose and fructose into the blood.

- Digestion and absorption are nevertheless essentially complete by the end of the small intestine, thus isomaltulose is a fully available carbohydrate, i.e., it provides the same energy as other available carbohydrates like sucrose or maltodextrin: $4 \mathrm{kcal} / \mathrm{g}$.

- The slower yet complete digestion and absorption are reflected in the blood glucose response in the form of a slower and overall lower rise in blood glucose levels.

- The lower blood glucose response is associated with less insulin release. 
In this way, isomaltulose differs from sucrose, glucose syrups (starch hydrolysates) and other traditional readily available carbohydrates, which commonly show a rapid rise in blood glucose levels to high concentrations, followed by a high insulin release and an insulin-induced rapid return of blood glucose to baseline levels and below. Using the low glycaemic carbohydrate isomaltulose instead of traditionally more frequently used high glycaemic carbohydrates can reduce the postprandial blood glucose response of foods. Carbohydrate-based foods with reduced glycaemic properties provide valuable alternatives that contribute to lower blood glucose excursions within a carbohydrate-based diet as recommended by nutritional guidelines.

Effect of isomaltulose on blood glucose and insulin levels: summary of human intervention studies

Numerous human intervention studies compared the blood glucose response of isomaltulose with that of sucrose or other traditional readily available carbohydrates. The majority of studies examined the corresponding insulin response in parallel. A range of ages from 18 years to elderly was covered and studies were conducted in various regions of the world (Europe, Asia, North America and Australia). Intake levels were between $75 \mathrm{~g}$ and $10 \mathrm{~g}$, mainly in beverage-type applications, although other matrices were measured as well.

Isomaltulose showed a lower blood glucose response than equal amounts of sucrose or maltodextrin in all of these studies and, in parallel, a lower and reduced insulin response.

Most of the studies were conducted in healthy adults of normal weight. The study in overweight to obese adults [10], the study in overweight and obese individuals with impaired glucose tolerance [11] and the study with diabetic individuals predominantly treated by diet alone [12] all confirm the lower effect of isomaltulose on blood glucose and insulin concentrations in comparison with sucrose for these population groups. Lower blood glucose responses with isomaltulose in adults with diabetes mellitus have also been observed in the study by Liao et al. [13] (non-insulin-dependent type 2 diabetes mellitus, in comparison to sucrose) and in the studies by West et al. [14] and Bracken et al. [15] (type 1 diabetes mellitus, in comparison to dextrose).

Considering the prevalence of impaired glucose tolerance and diabetes mellitus in the general population, the specific population groups covered by the totality of these studies can be regarded as representative of the general population.

The low glycaemic and low insulinaemic properties of isomaltulose described above are associated with further related physiological benefits, demonstrated in a growing number of acute and longterm studies:

- Improved glucose supply: The slower glucose release and resulting lower rise in blood glucose levels are associated with a more balanced supply of glucose over a longer period of time - a "sustained energy" supply.

- Improved metabolic regulation: The lower insulin release after isomaltulose intake leads to less severe metabolic changes in the postprandial phase. The hormone insulin plays a key role in the metabolic regulation after food intake: being released upon rising blood glucose levels, insulin promotes the uptake of nutrients from the blood into cells and their subsequent storage. Moreover, it promotes the predominant use of carbohydrates and suppresses the utilisation of fat sources in energy metabolism. Whereas high insulin levels triggered by the consumption of medium to high glycaemic carbohydrates are associated with a more extensive switch towards nutrient storage and suppression of fat utilisation, isomaltulose provides carbohydrate energy in a more balanced way with less insulin release and, subsequently, less severe metabolic changes.

- Energy partitioning and fat utilisation: The lowering effect of isomaltulose on blood glucose and insulin levels leads to a higher fat utilisation in energy metabolism. In other words, isomaltulose promotes fat oxidation when consumed in place 
of readily available high glycaemic carbohydrates. This effect has been demonstrated for isomaltulose in several human intervention studies in different population groups including healthy [16] and overweight to obese adults $[10,11]$ as well as in trained athletes. It is somewhat unique to isomaltulose because of the limited availability of carbohydrates with low glycaemic properties and the fact that it does not equally apply to fructose because of its liver metabolism. Research with isomaltulose has even shown benefits of the lower and sustained glucose supply to the brain and the working muscle with subsequent benefits for mental and physical performance.

- Weight management and body composition: Longer-term feeding studies with isomaltulose or isomaltulose-containing diets in rats and mice indicated a significant effect of the nutritional properties of isomaltulose on body fat disposition and body weight [17-19]. Also, human studies have given initial indications of beneficial effects on less visceral fat accumulation with isomaltulose [20-22].

- Long-term blood glucose control: Some studies found positive effects of sugar replacement by isomaltulose on markers directly related to longer-term blood glucose control and insulin sensitivity such as glycated haemoglobin HbA1c and oral glucose tolerance or insulin resistance (HOMA-IR) [9,21-23].

- Cardiovascular risk: Effects of isomaltulose on cardiovascular risk markers have also been addressed in studies with subjects of impaired glucose control/diabetes and/or impaired lipid metabolism $[9,11,24]$. None of these studies found detrimental effects on blood lipids (including cholesterol and low-density lipoprotein cholesterol) or cardiovascular risk markers.

These are the reasons supporting the use of isomaltulose in subjects willing to lower their postprandial blood glucose response, taking advantage of the benefits in modern energy management and performance as well as the long-term benefits related to health and the prevention and management of glucose tolerance impairments.

\section{Improvement of the quality of physiological} carbohydrates by replacement of availlable carbohydrates with partially or non-availlable carbohydrates: reduction of glucose supply

While in the case of isomaltulose a reduction of the blood glucose response was achieved by the modification of glucose supply via a smart choice of low glycaemic alternatives (i.e., isomaltulose), the other aspect that needs to be highlighted follows the principle of replacing available carbohydrates with partially or non-available carbohydrates. This leads to a reduction of glucose supply and thus to lower glycaemic and insulinaemic responses. This approach is relevant for classical sugar replacers like isomalt or other polyols. It is also relevant for some dietary fibres, i.e., inulin including oligofructose where the replacement of sugars is technically feasible with excellent sensorial results. This means, at the same time, enrichment by dietary fibres, which is an additional benefit.

\section{Lower blood glucose response by reducing glucose supply via a cup-by-cup replacement of sucrose by isomalt or other polyols}

Isomalt is a hydrogenated carbohydrate, made from sugar. It is a low-digestible carbohydrate, i.e., the vast majority passes the small intestine and reaches the large intestine where it is fermented to shortchain fatty acids. It has a very low effect on blood sugar and does not trigger insulin release to any significant extent. If calculated as an available carbohydrate qualifying for a GI calculation, the GI would be 2 [25].

Isomalt and other polyols have a history of use in particular in diets for diabetics as this was the traditional target group interested in low glycaemic response food. The physiological properties of polyols are reviewed in Livesey [26]. Even the replacement of only $30 \mathrm{~g}$ of sucrose by $30 \mathrm{~g}$ of isomalt leads to a significant improvement of long-term blood glucose 
parameters like HbA1c and insulin resistance, as demonstrated in a 12-week human intervention study with type II diabetics $(n=31)$ [27].

Lower blood glucose response by reducing glucose supply via replacement with dietary fibres

Lowering the blood glucose profile by replacing highly available starches and sugars with non-available dietary fibres is a fibre enrichment of the diet at the same time. This means two desired beneficial interventions can be achieved simultaneously. Inulin and oligofructose, both inulin-type fructans and ingredients derived from the chicory plant, qualify for this replacement from both food application and physiological points of view. The final product will result in a lower rise in blood sugar [28]. In addition to the example of inulin-type fructans replacing sugars, maltodextrins can be replaced by resistant dextrin, starch by resistant starch, refined flour by whole grain flour, etc.

\section{Conclusion}

Carbohydrate quality (expressed by the potential effect on blood glucose rise) matters when a healthier diet is the goal. Digestibility, availability, speed of absorption and metabolic pathways are key influencers determining the quality of carbohydrates. The overall aim is to achieve a lower glycaemic diet. There are several options leading to good tasting and lower glycaemic food for the benefit of consumers in their attempt to achieve a healthier diet and fight the burden of non-communicable diseases in the long term.

Reducing the blood glucose response of available carbohydrates by modification of the glucose supply is an option that can be achieved by the processing technique (starch-containing food), by influencing the gastrointestinal passage (viscous fibres) and by a smart choice of ingredients: by going for naturally low glycaemic and slow-release carbohydrates (isomaltulose instead of sucrose or maltodextrin).

Replacing available carbohydrates with partially or non-available carbohydrates (i.e., reduction of glu- cose supply) is the other route. Dietary fibre enrichment and the use of traditional sugar replacers (polyols) are the choices for non-available carbohydrates. The consumer can expect continuous support from the food industry, with innovative carbohydrates, smart ingredient choices and a commitment to the development of lower glycaemic food for a healthier and prevention-oriented lifestyle.

\section{Conflict of interest}

Anke Sentko works for Beneo Institute.

\section{Human and Animal Rights}

This article does not contain any studies with human or animal subjects performed by any of the authors.

\section{References}

1. European Food Safety Authority (2010) EFSA sets European dietary reference values for nutrient intakes. News story 26 March. http://www.efsa.europa.eu/en/press/news/nda100326. htm. Accessed 19 November 2013

2. WHO/FAO (2003) Diet, nutrition and the prevention of chronic diseases. WHO Technical Report Series 916. WHO/FAO, Geneva

3. EU Commission Decision 2005/581/EC of 25th July 2005 authorising the placing on the market of isomaltulose as a novel food or novel food ingredient under Regulation (EC) no 258/97 of the European Parliament and of the Council. Official Journal of the European Union 29.7.2007, L199/90L199/91

4. (2011) EFSA Panel on Dietetic Products, Nutrition and Allergies (NDA); Scientific Opinion on the substantiation of health claims related to the sugar replacers xylitol, sorbitol, mannitol, maltitol, lactitol, isomalt, erythritol, D-tagatose, isomaltulose, sucralose and polydextrose and maintenance of tooth mineralisation by decreasing tooth demineralisation (ID 463, 464, 563, 618, 647, 1182, 1591, 2907, 2921, $4300)$, and reduction of post-prandial glycaemic responses (ID 617, 619, 669, 1590, 1762, 2903, 2908, 2920) pursuant to Article 13(1) of Regulation (EC) No 1924/2006. EFSA Journal 9(4):2076. [25 pp.]. doi:10.2903/j.efsa.2011.2076.

5. Siddiqui IR, Furgala B (1967) Isolation and characterization of oligosaccharides from honey. Part 1. J Apic Res 6(3):139149

6. Eggleston G, Grisham M (2003) Oligosaccharides in cane and their formation on cane deterioration. In: Eggleston G, Cote G (eds) Oligosaccharides in food and agriculture. ACS Symposium Series. ACS, Washington, DC, pp 211-232

7. Irwin WE, Sträter PJ (2001) Isomaltulose. In: O'Brien-Nabors L (ed.) Alternative sweeteners, 3rd edn. Marcel Dekker, New York, pp 413-421 
8. Sentko A, Bernard J (2012) Isomaltulose. In: O'Brien-Nabors L (ed.) Alternative sweeteners, 4th edn. Taylor \& Francis, Boca Raton, FL, pp 423-438

9. Holub I, Gostner A, Theis S, Nosek L, Kudlich T, Melcher R et al (2010) Novel findings on the metabolic effects of the low glycaemic carbohydrate isomaltulose (Palatinose). Br J Nutr 103(12):1730-1737

10. van Can JG, Ijzerman TH, van Loon LJ, Brouns F, Blaak EE (2009) Reduced glycaemic and insulinaemic responses following isomaltulose ingestion: implications for postprandial substrate use. Br J Nutr 102(10):1408-1413

11. König D, Theis S, Kozianowski G, Berg A (2012) Postprandial substrate use in overweight subjects with the metabolic syndrome after isomaltulose (Palatinose) ingestion. Nutrition 28(6):651-656

12. Kawai K, Yoshikawa H, Murayama Y, Okuda Y, Yamashita K (1989) Usefulness of palatinose as a caloric sweetener for diabetic patients. Horm Metab Res 21(6):338-340

13. Liao ZH, Li YB, Yao B, Fan HD, Hu G, Weng JP (2001) The effects of isomaltulose on blood glucose and lipids for diabetic subjects. Acta Nutrimenta Sinica 23(4):373-375

14. West DJ, Morton RD, Stephens JW, Bain SC, Kilduff LP, Luzio $S$ et al (2011) Isomaltulose improves postexercise glycemia by reducing $\mathrm{CHO}$ oxidation in T1DM. Med Sci Sports Exerc 43(2):204-210

15. Bracken RM, Page R, Gray B, Kilduff LP, West DJ, Stephens JW et al (2012) Isomaltulose improves glycemia and maintains run performance in type 1 diabetes. Med Sci Sports Exerc 44(5):800-808

16. Arai H, Mizuno A, Sakuma M, Fukaya M, Matsuo K, Muto $K$ et al (2007) Effects of a palatinose-based liquid diet (Inslow) on glycemic control and the second-meal effect in healthy men. Metabolism 56(1):115-121

17. Sato K, Arai H, Miyazawa Y, Fukaya M, Uebanso T, Koganei $\mathrm{M}$ et al (2008) Palatinose and oleic acid act together to prevent pancreatic islet disruption in nondiabetic obese Zucker rats. J Med Invest 55(3-4):183-195

18. Arai H, Mizuno A, Matsuo K, Fukaya M, Sasaki H, Arima H et al (2004) Effect of a novel palatinose-based liquid balanced formula (MHN-01) on glucose and lipid metabolism in male Sprague-Dawley rats after short- and long-term ingestion. Metabolism 53(8):977-983
19. Fujiwara T, Naomoto Y, Motoki T, Shigemitsu K, Shirakawa $\mathrm{Y}$, Yamatsuji T et al (2007) Effects of a novel palatinose based enteral formula (MHN-01) carbohydrate-adjusted fluid diet in improving the metabolism of carbohydrates and lipids in patients with esophageal cancer complicated by diabetes mellitus. J Surg Res 138(2):231-240

20. Yamori Y, Mori H, Mori M, Kashimura J, Sakamua T, Ishikawa PM et al (2007) Japanese perspective on reduction in lifestyle disease risk in immigrant Japanese Brazilians: a double-blind, placebo-controlled intervention study on palatinose. Clin Exp Pharmacol Physiol 34:S5-S7

21. Okuno M, Kim MK, Mizu M, Mori M, Mori H, Yamori Y (2010) Palatinose-blended sugar compared with sucrose: different effects on insulin sensitivity after 12 weeks supplementation in sedentary adults. Int J Food Sci Nutr 61(6):643-651

22. Oizumi T, Daimon M, Jimbu Y, Kameda W, Arawaka N, Yamaguchi $\mathrm{H}$ et al (2007) A palatinose-based balanced formula improves glucose tolerance, serum free fatty acid levels and body fat composition. Tohoku J Exp Med 212(2):91-99

23. Sakuma M, Arai H, Mizuno A, Fukaya M, Matsuura M, Sasaki $\mathrm{H}$ et al (2009) Improvement of glucose metabolism in patients with impaired glucose tolerance or diabetes by longterm administration of a palatinose-based liquid formula as a part of breakfast. J Clin Biochem Nutr 45(2):155-162

24. Brunner S, Holub I, Theis S, Gostner A, Melcher R, Wolf P et al (2012) Metabolic effects of replacing sucrose by isomaltulose in subjects with type 2 diabetes: a randomized double-blind trial. Diabetes Care 35(6):1249-1251

25. Sydney University. http://www.glycemicindex.com/foodSearch.php. Accessed 17 November 2013

26. Livesey G (2003) Health potential of polyols as sugar replacers, with emphasis on low glycaemic properties. Nutr Res Rev 16:163-191

27. Holub I, Gostner A, Hessdorfer S, Theis S, Bender G, Willinger B et al (2009) Improved metabolic control after 12week dietary intervention with low glycaemic isomalt in patients with type 2 diabetes mellitus. Horm Metab Res 41(12):886-892

28. Meyer D (2007) Inulin for product development of low GI products to support weight management. In: Salovaara $\mathrm{H}$, Gates F, Tenkanen M (eds) Dietary fibre components and functions. Academic, Wageningen, pp 257-269 